\title{
Whole Body MRI Intensity Standardization
}

\author{
Florian Jäger ${ }^{1}$, László Nyúl ${ }^{1}$, Bernd Frericks ${ }^{2}$, \\ Frank Wacker ${ }^{2}$ and Joachim Hornegger ${ }^{1}$ \\ ${ }^{1}$ Institute of Pattern Recognition, University of Erlangen, \\ \{jaeger,nyul,hornegger\}@informatik.uni-erlangen.de \\ ${ }^{2}$ Department for Radiology and Nuclear Medicine, \\ Charité, Campus Benjamin Franklin, Berlin, \\ \{bernd.frericks,frank.wacker\}@charite.de
}

\begin{abstract}
A major problem of segmentation of magnetic resonance images is that intensities are not standardized like in computed tomography. This article deals with the correction of inter volume intensity differences that lead to a missing anatomical meaning of the observed gray values. We present a method for MRI intensity standardization of whole body MRI scans. The approach is based on the alignment of a learned reference and the current histogram. Each of these histograms is at least 2-d and represents two or more MRI sequences (e.g., T1- and T2-weighted images). From the matching a non-linear correction function is gained which describes a mapping between the intensity spaces and consequently adapts the image statistics to a known standard. As the proposed intensity standardization is based on the statistics of the data sets only, it is independent from spatial coherences or prior segmentations of the reference and newly acquired images. Furthermore, it is not designed for a particular application, body region or acquisition protocol. The method was evaluated on whole body MRI scans containing data sets acquired by T1/FL2D and T2/TIRM sequences. In order to demonstrate the applicability, examples from noisy and pathological image series acquired on a whole body MRI scanner are given.
\end{abstract}

\section{Introduction}

For magnetic resonance imaging no protocol dependent intensity standard, like the Hounsfield units in computed tomography, is available due to magnetic field inhomogeneities in both $B_{0}$ and $\mathrm{RF}$ excitation fields. One type of variation is that intensities of the same tissue class differ throughout a single volume. In order to deal with that problem, a variety of algorithms for bias field correction were developed in the last decade. However, these methods do not solve the other type of problem: a certain measured intensity cannot be associated with a tissue class. The distinction of both kinds of variations is illustrated in Figure 1. For segmentation, a missing protocol dependent standard intensity scale has the disadvantage that for every new suspect an individual training of the used (statistical) model has to be performed. For this reason the clinical applicability of many algorithms is low due to runtime restrictions. Furthermore, visualization 
Fig. 1. The distinction of both types of variations (inter and intra scan inhomogeneities). The first image shows the original FL2D scan of a patient. The second image shows the same slice after gain field correction. In the third image a threshold of 580 is applied to the gain field corrected slice. The last image shows a FL2D scan of another patient after gain field correction with the same threshold applied
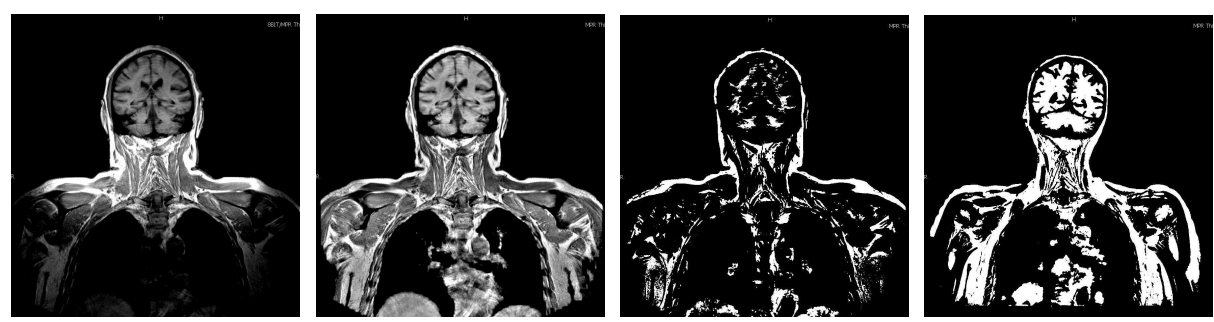

systems cannot use standard presets (e.g., transfer functions) to visualize certain organs or tissue classes. The settings have to be adjusted for every single scan. Hence, a second class of approaches dealing with inter-scan intensity standardization was developed by several authors. State-of-the-art algorithms, generally, standardize the observed intensities using a single image at a time and ignore spatially adjacent images. For many applications this is sufficient, because in many regions of the body a gray value in one image is associated with exactly one intensity in another sequence (e.g., the brain). In general, however, this is not the case. The algorithm presented in this article utilizes all acquired images for intensity standardization. With that, it is possible to separately correct tissue classes that have the same intensity in one image but can be distinguished using more data sets. Furthermore, the introduced approach does not rely on any assumptions about the shape of the joint histograms used. Thus the method is completely independent from the application, region of interest (brain, thorax, pelvis, etc.), scanning protocol (e.g., T1-, T2-weighted) as long as there are learned histograms available for the task.

In [1], a 1D histogram matching approach was presented. First, they detected some landmarks (percentiles, modes, ...) on the template and the reference histogram, matched them and finally interpolated linearly between the detected locations. Pierre Hellier presented in [2] a correction method for MRI brain images that estimates a mixture of Gaussians that approximates the histogram first. Then he computes a polynomial correction function that aligns the mean intensities of the tissues. A multiplicative correction field is estimated in [3], that adapts the intensity statistics of an acquired MR volume to a previously created model. This is achieved by minimizing the Kullback-Leibler divergence between the model and the template intensity distribution. In [4] a method including spatial information between the reference and the template image is presented. In order to match the images a non-linear registration algorithm was used. On the aligned images a scalar multiplicative correction weight is computed. How intensity standardization and bias correction influence each other is evaluated 
Fig. 2. Schematic illustration of the intensity standardization. First, from the reference images a reference joint histogram is created. This is the training component of the approach. Then from the current MRI images a joint histogram is generated. In the next step these histograms are non-rigidly registered. Using the gained transformation function, the current images are standardized

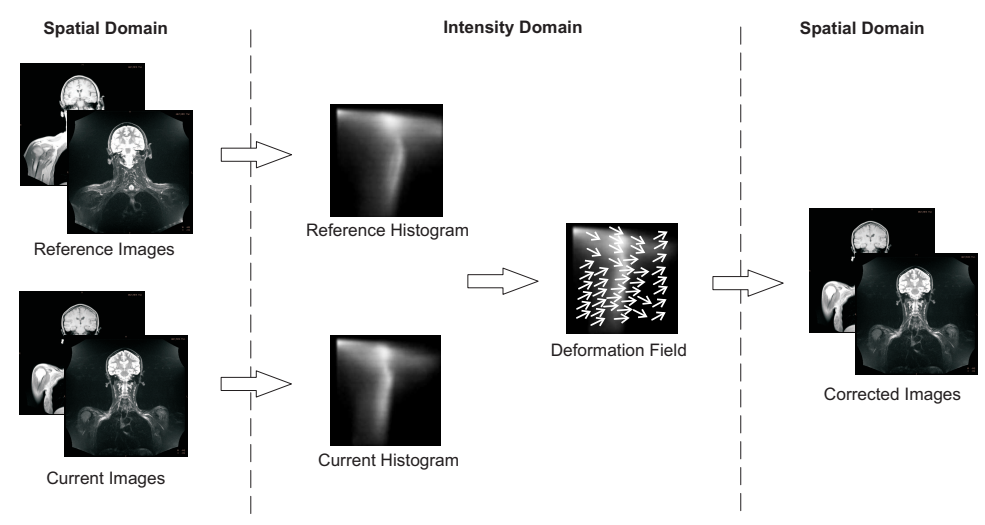

in [5]. The authors conclude that both steps are necessary but the correction of inhomogeneities has to be done beforehand.

\section{Methods}

The goal of the intensity standardization approach is to find a mapping between the intensities of a set of images $U=\left(U_{1}, U_{2}, \ldots, U_{n}\right)$, where $n$ is the number of images and a reference set of images $R=\left(R_{1}, R_{2}, \ldots, R_{n}\right)$ so that an intensity vector $i \in \mathcal{I}^{n}$ describes the same tissue class in both sets with $\mathcal{I}^{n}$ being the intensity space of the image sets [6]. The main idea of this contribution is that this mapping can be approximated by the minimization of the distance between the joint relative histograms of the two sets of images. The required relative joint histograms have a dimensionality of $n$. The domain is $\mathbb{R}^{n}$. In general, however, it can be scaled to $[0,1]^{n}$ due to the limited number of gray values observed. The relative joint histograms of the two tuples will usually never be equal (at least for real data sets) as the volume of the same tissue class in the image set $U$ and $R$ differs for inter- as well as for intra-patient measurements (e.g., partial volume averaging effects, positioning of the patient). Thus the search for a mapping between the intensity spaces is equivalent to finding the deformation between the relative joint histograms $\mathcal{H}(R)$ and $\mathcal{H}(U)$ so that they are closest with respect to a given distance measure. If the joint histograms are treated as images, this task can be viewed as non-rigid image registration [7].

The used whole body MRI data sets are very large, thus the influence of small local structures on the appearance of the histogram is very low. For this reason we split the volume in $K$ sub volumes. These sub volumes are than corrected separately. However, in order to guarantee a common gray level standard, the 
Fig. 3. First row: head region of the reference image (FL2D), the reference image (TIRM), the template image (FL2D) and the template image (TIRM). Second row: Thresholded images, FL2D protocol (Reference, Template, Result). The threshold for the binarization of all images is the same. The effect of the intensity standardization can be seen best in the brain area

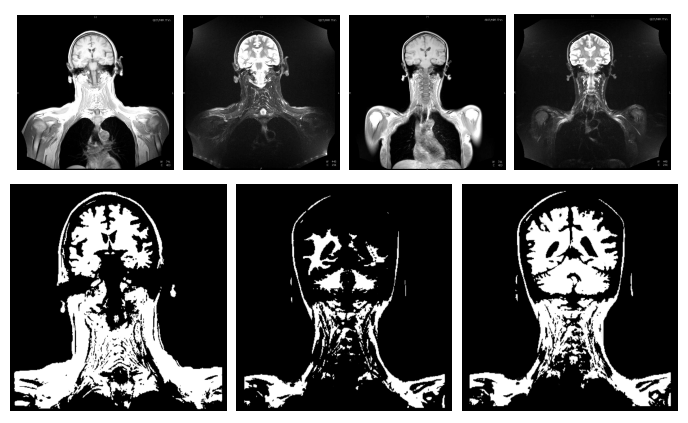

other $K-1$ histograms are used as additional regularizer. Consequently our new distance measure can be written as

$$
\mathcal{D}^{i}\left[\mathcal{H}(R), \mathcal{H}(U) ; \boldsymbol{u}^{i}\right]=\sum_{k=1}^{K} a_{i, k} \mathcal{D}\left[\mathcal{H}\left(R^{k}\right), \mathcal{H}\left(U^{k}\right) ; \boldsymbol{u}^{i}\right], \quad \sum_{k=1}^{K} a_{i, k}=1
$$

where $i$ is the current sub volume and $a_{i, k}$ is the influence of the force of sub volume $k$ in the context of the standardization of block $i$. $\mathcal{D}$ is a distance measure like sum of squared differences or mutual information. $R^{k}$ and $U^{k}$ are the $k-t h$ sub volumes and $\boldsymbol{u}^{i}$ is the current deformation field.

The result of the optimization is the transformation $T: \mathbb{R}^{n} \mapsto \mathbb{R}^{n}$. In the case of the registration of multi-dimensional relative joint histograms, it describes how to transform the gray values of one set of images $U$ such that its intensity distribution best matches the reference distribution, with respect to the used distance measure and smoother. Here a curvature based smoother was used. Thus the intensity standardization can be done by $\boldsymbol{i}_{\text {corr }}=T\left(\boldsymbol{i}_{\text {orig }}\right)$.

\section{Results}

All data sets were acquired on a Siemens Avanto 1.5 T whole body MRI scanner. The TIRM images had a resolution of $512 \times 512 \times 30$ (each block) with $0.98 \mathrm{~mm}^{2}$ and $5.5 \mathrm{~mm}$ slice thickness and $\mathrm{TE}=83$ and $\mathrm{TR}=1660$ and the FL2D images had a resolution of $512 \times 410 \times 30$ (each block) with $0.98 \mathrm{~mm}^{2}$ and $5.5 \mathrm{~mm}$ slice thickness and $\mathrm{TE}=4.7$ and $\mathrm{TR}=291$. The size of the composed whole body images was $542 \times 1746 \times 20$ for both protocols. Only the composed volumes were used for the experiments. All images were acquired in clinical routine. In total ten whole body MRI data sets were used for evaluation. 
We divided the volumes in $K=5$ blocks along the $y$-direction. Thus each block had a size of $542 \times 350 \times 20$. The size of a joint histogram was $128 \times 128$ pixel. The registration of one histogram took about one second. Using these settings, the overall computational time for the standardization was about seven seconds.

The difference $\mu_{d}$ of the mean intensities of the data sets was chosen as quality measure. For the TIRM images this resulted in a difference of $\mu_{d}=6.4$ before and $\mu_{d}=3.2$ after the standardization. As the TIRM images do not have many visible structures in the legs, the histograms are very blurred in these regions. Thus no reliable registration is possible. After removing these regions (block four and five) from the evaluation we got a difference of $\mu_{d}=7.0$ before and $\mu_{d}=2.3$ after standardization. Figure 3 illustrates the effect of the standardization.

\section{Discussion}

In this article we present a new method for MRI intensity standardization of whole body MRI scans. In contrast to most of the previously published methods, the proposed approach is independent from any prior knowledge about the structure of the data sets and it relies on the image statistics only. Hence, there is no prior registration or segmentation of the data sets necessary. Furthermore, the method is independent from the application, body region and imaging protocol, if learned joint histograms are available. The most important improvement is that the proposed method utilizes all acquired images jointly and does not perform the standardization of the images separately. However, if the image statistics are too different, the obtained results may not be satisfying. This is the case, for instance, for volumes disturbed by a strong bias field. This yields blurred histograms and thus no reliable registration is possible anymore.

\section{References}

1. Nyúl LG, Udupa JK, Zhang X. New variants of a method of MRI scale standardization. IEEE Trans Med Imaging 2000;19(2):143-150.

2. Hellier P. Consistent Intensity Correction of MR images. In: Procs ICIP. vol. 1; 2003. 1109-1112.

3. Weisenfeld NI, Warfield SK. Normalization of joint image intensity statistics in MRI using the Kullback-Leibler divergence. In: Procs IEEE ISBI. vol. 1; 2004. 101-104.

4. Schmidt M. A Method for Standardizing MR Intensities between Slices and Volumes. University of Alberta; 2005.

5. Madabhushi A, Udupa JK. Interplay between intensity standardization and inhomogeneity correction in MR image processing. IEEE Trans Med Imaging $2005 ; 24(5): 561-576$.

6. Jäger F, Deuerling-Zheng Y, Frericks B, Wacker F, Hornegger J. A new method for MRI intensity standardization with application to lesion detection in the brain. Procs VMV 2006; 269-276.

7. Modersitzki J. Numerical Methods for Image Registration. Oxford University Press, Oxford New York; 2004. 